\title{
Timing-Synchronization Analysis for IEEE 802.11a Wireless LANs in Frequency-Nonselective Rician Fading Environments
}

\author{
Kun-Wah Yip, Member, IEEE, Yik-Chung Wu, Student Member, IEEE, and Tung-Sang Ng, Fellow, IEEE
}

\begin{abstract}
This paper derives and computes the probability of synchronization failure $P_{\text {fail }}$ for IEEE 802.11a wireless LANs on frequency-flat Rician fading channels. For a frequency offset within $\pm 232 \mathrm{kHz}$, it is shown that its effect on the synchronization performance is minor. The $E_{\mathrm{ds}} / N_{0}$ ratios required to achieve $P_{\text {fail }}=10^{-3}$ and $10^{-4}$ are computed, where $E_{\mathrm{ds}}$ is the data-symbol energy. We find that $E_{\mathrm{ds}} / N_{0}$ ratios over $20 \mathrm{~dB}$ are generally required for channels with Rician factors $K \leq 6 \mathrm{~dB}$. In particular, $E_{\mathrm{ds}} / N_{0}$ ratios that yield $P_{\text {fail }}=10^{-4}$ exceed $30 \mathrm{~dB}$ for $K \leq 4 \mathrm{~dB}$.
\end{abstract}

Index Terms-Frequency-nonselective channel, IEEE 802.11a, orthogonal frequency division multiplexing (OFDM), probability of synchronization failure, Rician fading, sliding correlator, timing synchronization, wireless local area network (LAN).

\section{INTRODUCTION}

$\mathbf{I}$ EEE 802.11a wireless local area networks (LANs) support packetized data transmission at a high rate up to $54 \mathrm{Mb} / \mathrm{s}$ [1]. In packetized data transmission, data symbols cannot be demodulated if the receiver cannot establish timing synchronization with the received signal. The probability of synchronization failure is therefore one of the important parameters in the determination of packet retransmission probabilities. In this paper, we derive and compute the probability of synchronization failure for IEEE 802.11a wireless LANs operating on frequency-flat Rician fading channels. Before proceeding further, we provide a justification on the consideration of frequencynonselective fading channels in the analysis.

IEEE 802.11a wireless LANs operate in the 5-GHz band with a signal bandwidth that is about $20 \mathrm{MHz}$ [1]. Usually, they are used for communication inside buildings. In many propagation studies, e.g., [2] and [3], the measured coherence bandwidths of indoor channels were smaller than $20 \mathrm{MHz}$ so that the channels were frequency selective. These studies considered a real-

Manuscript received August 1, 2001; revised July 17, 2002, January 9, 2003, and February 18, 2003; accepted February 19, 2003. The editor coordinating the review of this paper and approving it for publication is P. F. Driessen. This work was supported by the Hong Kong Research Grants Council and by the University Research Committee of The University of Hong Kong, Hong Kong. This paper was presented in part at the IEEE Wireless Communications and Networking Conference (WCNC), New Orleans, LA, March 16-20, 2003.

K.-W. Yip and T.-S. Ng are with the Department of Electrical and Electronic Engineering, The University of Hong Kong, Hong Kong (e-mail: kwyip@eee.hku.hk; tsng@eee.hku.hk).

Y.-C. Wu was with the Department of Electrical and Electronic Engineering, The University of Hong Kong, Hong Kong. He is now with the Department of Electrical Engineering, Texas A\&M University, College Station, TX 778433128 USA (e-mail: ycwu@ee.tamu.edu).

Digital Object Identifier 10.1109/TWC.2004.825372 istic situation that the transmitter and receiver were separated generally by a distance of more than $10 \mathrm{~m}$. However, wireless LANs can also be deployed in environments where the access point and the mobile station are separated by a shorter distance, e.g., inside small rooms. It is possible that the delay spread is small and the coherence bandwidth is greater than $20 \mathrm{MHz}{ }^{1}$ In [4], measurement results have shown that coherence bandwidths recorded in a university laboratory were around $40 \mathrm{MHz}$ for a correlation level of 0.5 , indicating that the channels were frequency nonselective. In [5], results measured in a residential room have indicated that in most cases the root-mean square (rms) delay spreads were less than $10 \mathrm{~ns}$. Using the result that the coherence bandwidth is approximately equal to the reciprocal of five times of the rms delay spread [6, p. 164], one can model the channel considered in [5] as a frequency-nonselective channel. Also notice that there is increasing interest in the use of wireless LANs for in-home entertainment applications that involve multimedia data distribution [7]. Thus, it is relevant to consider frequency-nonselective fading channels in the analysis of the synchronization performance.

Theoretical characterization for the case of frequency-nonselective fading channels is also useful in shortening the design time of wireless LAN systems intended for frequency-selective fading channels. In [8], the authors investigated the synchronization performance of IEEE 802.11a wireless LANs operating on frequency-selective Rician fading channels by simulation. It is noted that while simulation takes a long time to yield a result on the synchronization performance, theoretical analysis for the case of frequency-selective fading channels is very difficult, if not impossible. Using the theoretical result of this work enables system designers to initially estimate the synchronization performance rapidly for different options of system configurations at an early design stage. When a good candidate configuration is identified, system designers can verify its goodness by evaluating the actual performance for frequency-selective fading channels through simulation. We mention that one application of the present work to speed up the design cycle was the determination of correlator coefficients for multiplierless correlators used in synchronization circuits, with an objective that the synchronization performance was not degraded when compared to that of nonmultiplierless correlators [9]. The best set of correlator coefficients was selected based on computer search. Since it was required to evaluate the synchronization perfor-

\footnotetext{
${ }^{1}$ Notice that the delay spread depends not only on the separation between the access point and the mobile station but also on other factors such as the scatterer distribution, room size, and building materials.
} 
mance for a large number of different combinations of correlator coefficients, using the formulas derived from the present work ${ }^{2}$ instead of entirely resorting to simulation greatly reduced the design time.

The contribution of this paper is elaborated as follows. IEEE 802.11a wireless LANs employ orthogonal frequency-division multiplexing (OFDM) as the transmission technique. Although timing synchronization for OFDM systems has been investigated in a number of works [10]-[16], these works have only considered correlating the received signal with a copy of itself time delayed by a given offset. Results of these works are generally not applicable to IEEE 802.11a wireless LANs, in which synchronization is accomplished by correlating the received signal with a known reference waveform, which is the pilot OFDM symbol of the preamble. OFDM timing synchronization using pilot symbols and correlation techniques has been studied for multipath fading channels in [17]-[19] through simulation. The theoretical performance has been analyzed for Gaussian channels in [20]. Since the system configurations and pilot symbol assignments considered in these works are different from those specified in the IEEE 802.11a specifications [1], results therein cannot provide insights into the synchronization performance of IEEE 802.11a wireless LANs. In this paper, a new expression for computing the probability of synchronization failure for IEEE 802.11a wireless LANs on frequency-flat Rician fading channels is derived. Rician distribution is considered because it has been shown to be appropriate in modeling the fading characteristic of indoor radio channels [21]-[23].

The rest of the paper is organized as follows. An overview of timing-synchronization aspects for IEEE 802.11a wireless LANs is provided in Section II. After describing the system model in Section III, we derive the probability of synchronization failure in Section IV. A discussion of the synchronization performance is given in Section V. Finally, Section VI concludes the paper.

\section{TIMING Synchronization IN IEEE 802.11a WIRELESS LANS}

A $16-\mu$ s preamble having a structure shown in Fig. 1 is inserted at the beginning of each data packet. The preamble is divided into two subpreambles. The first one consists of ten identical short OFDM symbols each having a length of $800 \mathrm{~ns}$. The second one comprises two long OFDM symbols each of length $3.2 \mu$ s preceded by a 1.6- $\mu$ s cyclic prefix. The first subpreamble is used for initial detection of the signal, automatic gain control, diversity selection, coarse frequency-offset compensation, and timing synchronization. The second one enables channel estimation and fine frequency-offset compensation.

It is known that frequency-offset estimation can be accomplished by performing two fast Fourier transforms (FFTs) on the portion of signal comprising two repeated symbols and then measuring the phase rotation [24]. Since the first subpreamble is a repetition of identical symbols, the signal is periodic with a period of $800 \mathrm{~ns}$. The receiver can choose any $1.6-\mu \mathrm{s}$ window

\footnotetext{
${ }^{2} \mathrm{~A}$ slight modification of the formulas given in this paper was required in order to take into account the effect of using different correlator coefficients.
}

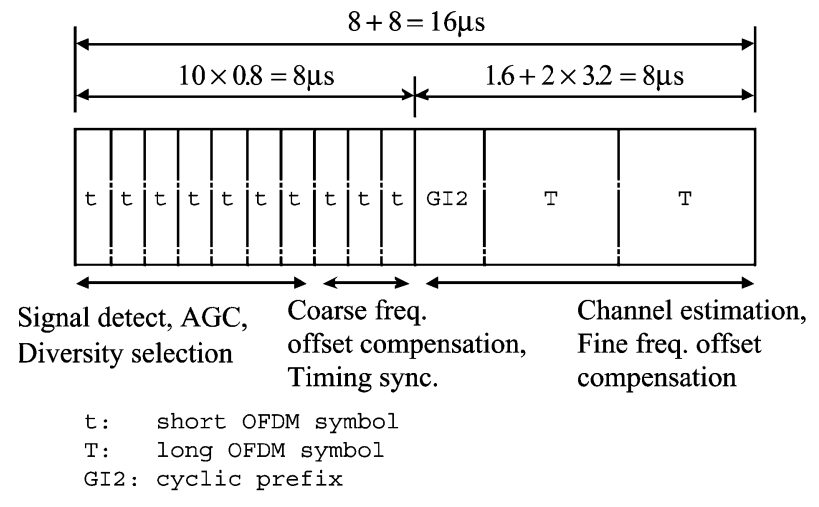

Fig. 1. Preamble structure of an IEEE 802.11a data packet.

to perform FFTs and need not know the exact timing of symbol boundaries. On the other hand, it is required to detect the end of the first subpreamble. This time reference enables the receiver to locate the time instant in the second subpreamble at which the FFT window for fine frequency-offset compensation begins. To detect the end of the first subpreamble, the receiver can correlate the received signal with the short OFDM symbol. The presence of a correlation peak indicates that the first subpreamble has not passed while the absence of an expected correlation peak is an indication that the current time position is at $800 \mathrm{~ns}$ in the second subpreamble. A sliding correlator, which produces correlation results at a rate equal to the rate of incoming signal samples, is required because the receiver needs to perform rapid synchronization in a short time. A sampling rate of $20 \mathrm{MHz}$ is recommended in the digital implementation of the correlator. ${ }^{3}$ This sampling rate is suggested in [1] originally for the convenience in the implementation of OFDM demodulators.

Notice that in the specifications [1] only a duration of three short OFDM symbols $(3 \times 800 \mathrm{~ns})$ is allocated for performing both coarse frequency-offset compensation and timing synchronization. As frequency-offset compensation requires a time of two OFDM symbols [24] plus computation time for offset calculation, it does not appear practical to perform timing synchronization after the received signal is frequency-offset compensated. It follows that both processes are simultaneously carried out. A large frequency offset may be present during correlation. It is required that the deviation of the transmitted-signal center frequency is within $\pm 20 \mathrm{ppm}$ [1]. Assume that the receiver oscillator also has a frequency uncertainty of $\pm 20 \mathrm{ppm}$ and that the operating frequency is $5.8 \mathrm{GHz}$ [1]. The frequency offset is within $\pm 232 \mathrm{kHz}$.

\section{SYNCHRONIZATION MODEL}

The OFDM signal specified in the standard [1] comprises 52 subcarriers numbered from -26 to +26 with no signal transmission at the zeroth subcarrier. All subcarriers are utilized to generate the long OFDM symbol. For the short one, transmission is confined to the subcarriers whose numbers are multiples

\footnotetext{
${ }^{3}$ Note that the correlator only needs to perform 16 multiplications to produce a correlation result every $50 \mathrm{~ns}$. The implementation complexity involved is generally not demanding, and the use of a sliding correlator for timing synchronization is realistic.
} 
of four, so that only 12 subcarriers are used. The complex envelope of the preamble $s(t)$ is given by [1]

$$
\begin{aligned}
& s(t) \\
& =\sqrt{2 P} \times\left[\sum_{k=-26}^{26} B_{k} e^{j 2 \pi k \Delta_{f} t} \operatorname{rect}\left(0, T_{p 1}\right)\right. \\
& \left.+\sum_{k=-26}^{26} C_{k} e^{j 2 \pi k \Delta_{f}\left(t-T_{p 1}-T_{G I 2}\right)} \operatorname{rect}\left(T_{p 1}, T_{p 1}+T_{p 2}\right)\right]
\end{aligned}
$$

where $T_{p 1}=8 \mu \mathrm{s}$ and $T_{p 2}=8 \mu \mathrm{s}$ are the lengths of the first and second subpreambles, respectively, $T_{G I 2}=1.6 \mu \mathrm{s}$ is the length of the cyclic prefix of the second subpreamble, $\operatorname{rect}\left(t_{1}, t_{2}\right)$ is the rectangular window with unity gain over time $t_{1}$ to $t_{2}, P$ is the signal power, $\Delta_{f}=312.5 \mathrm{kHz}$ is the subcarrier spacing, and $B_{k}=52^{-1 / 2} S_{k}$ and $C_{k}=52^{-1 / 2} L_{k}$ are pilot symbols used to generate the short and long OFDM symbols, respectively. $S_{k}$ and $L_{k}$ are detailed in [1, pp. 12-13]. We mention that $B_{0}=$ $C_{0}=0$ and $B_{k}=0$ for $k$ not a multiple of four. The signal model given by (1) is slightly different from the one specified in the standard [1] in that raised-cosine windows rather than rectangular ones should be used. The assumption of rectangular windows is to simplify analysis. Raised-cosine windows were used in the simulation to verify the analytical result.

The complex envelope of the received signal after RF filtering $r(t)$ is given by

$$
r(t)=\eta(t)+e^{j 2 \pi f_{\text {off }} t} \xi \cdot s(t)
$$

where $\eta(t)$ is the filtered complex-Gaussian noise, $f_{\text {off }}$ is the frequency offset, and $\xi$ is a complex path gain of the frequencynonselective Rician fading channel. The noise power is computed by

$$
\frac{1}{2} E\left\{|\eta(t)|^{2}\right\}=N_{0} W
$$

where $N_{0}$ is the one-sided noise power spectral density of additive white Gaussian noise (AWGN) and $W$ is the noise bandwidth of the RF filter. Since the signal energy is concentrated within a bandwidth of $18 \mathrm{MHz}$ [1], we consider that $W=18 \mathrm{MHz}$. Let

$$
v=|\xi|^{2}
$$

Assume that the channel is of unity gain. The probability density function of $v$ is given by [25, ch. 2-1-4]

$$
p(v)=(K+1) I_{0}(2 \sqrt{K(K+1) v}) e^{-(K+1) v-K}, \quad v \geq 0
$$

where $K$ is the Rician factor of the channel. The received signal is digitized at a sampling rate of $1 / T_{\text {sam }}$ samples per second with $T_{\text {sam }}^{-1}=20 \mathrm{MHz}$. The $n$th signal sample $r_{n}$ is given by

$$
r_{n}=r\left(n T_{\mathrm{sam}}+\tau_{\mathrm{sam}}\right)
$$

where $\tau_{\text {sam }}$ is the fractional time offset modeled by a uniform random variable over $\left[-(1 / 2) T_{\mathrm{sam}},(1 / 2) T_{\mathrm{sam}}\right]$. This fractional time offset is a result of asynchronism between the sampling clock and the received signal. It is possible that the noise components of $r_{n}$ s are correlated where the correlation depends on the frequency response of the RF filter and the sampling rate.
In the present work, since the filter bandwidth is approximately $20 \mathrm{MHz}$ and the sampling rate is also $20 \mathrm{MHz}$, we make a reasonable assumption that this correlation is negligible.

A sliding correlator is used to process the received signal samples. We denote $T_{\mathrm{ss}}=800 \mathrm{~ns}$ as the length of the short OFDM symbol. The correlator output obtained at time $n T_{\text {sam }}+\tau_{\text {sam }}$ is computed by

$$
\Xi_{n}=\frac{1}{\sqrt{2 P}} \sum_{m=1}^{M} r_{n-M+m} g_{m}^{*}
$$

where

$$
M=T_{\mathrm{ss}} / T_{\mathrm{sam}}=16
$$

is the number of signal samples involved in correlation, and the weights

$$
g_{m}=\sum_{k=-26}^{26} B_{k} e^{j 2 \pi k \Delta_{f} m T_{\mathrm{sam}}}, \quad m=1,2, \cdots, M
$$

constitute a short OFDM symbol. The synchronization procedure is as follows. The time location of the correlation peak and its $\left|\Xi_{n}\right|^{2}$ value are identified by searching for the maximum value of $\left|\Xi_{n}\right|^{2}$ over the obtained correlation results. After the correlation peak is located, the next $M$ th correlation result is compared with the maximum value of $\left|\Xi_{n}\right|^{2}$ to determine if another correlation peak is present. The search for expected correlation peaks continues until an absence is identified, in which the second subpreamble is entered. Note that a new correlation result is generated every sampling instant so that the maximum value of $\left|\Xi_{n}\right|^{2}$ and the corresponding time location are regularly updated. Let $\mu_{i}$ and $n_{i}$ be the maximum value of $\left|\Xi_{n}\right|^{2}$ and the corresponding time location, respectively, recorded at the $i$ th sampling instant but excluding the current correlation result. They are given by

$$
\mu_{i}=\max _{n<i}\left|\Xi_{n}\right|^{2}
$$

and

$$
n_{i}=\underset{n<i}{\arg \max }\left|\Xi_{n}\right|^{2} .
$$

The end of the first subpreamble is declared at the sampling instant $i-M$ if $i-n_{i}$ is a multiple of $M$ and

$$
\beta \mu_{i}>\left|\Xi_{i}\right|^{2}
$$

is satisfied, where $\beta \in(0,1)$ is a given threshold value.

\section{Probability of SynChronization FAILURE}

In practical receivers, signal samples that can be used to compute valid correlation results are available only after the amplifier gain of the automatic gain control (AGC) circuit is settled. In this paper, we follow the suggestion of the specifications [1] that the first seven short OFDM symbols are allocated for the settling of AGC amplifier gain, while the last three are used for timing synchronization. Implicitly, it is required that the receiver can roughly locate the end time of the seventh short OFDM symbol. This requirement can be fulfilled, in general, as the receiver can usually detect the arrival of signal (i.e., initial detection of the signal) within a small, bounded delay. Since signal samples that can be used to compute correlation results roughly start from the eighth short OFDM symbol, it is 
expected that correlation peaks occur at $n=8,9$, and $10 M$. In practice, however, the locations of correlation peaks are not deterministic. They may shift from these expected locations due to the presence of noise (which magnifies its effect on the peak locations if the channel is in deep fade), the possible presence of fractional sampling-time offset $\tau_{\text {sam }}$, and the frequency offset $f_{\text {off }}$, etc. The nondeterministic nature of the peak locations makes the analysis of the timing-synchronization performance intractable. To make the analysis possible, we assume that the peak locations are deterministic and occur only at $n=8,9$, and $10 M$. It follows that the derived result only yields an approximation to the synchronization performance. The closeness of this approximation to the actual performance will be checked by simulation. At $n=11 \mathrm{M}$, the value of $\left|\Xi_{n}\right|^{2}$ drops significantly. An event of false alarm arises when (12) is satisfied for $n=9$ or $10 \mathrm{M}$. A miss occurs when (12) is not satisfied for $n=11 \mathrm{M}$. A synchronization failure is an event where either a false alarm or a miss occurs.

Expanding (6) by (2) and substituting the resultant expression into (7) yields

$$
\begin{aligned}
& \Xi_{q M} \\
& \quad= \begin{cases}\eta_{q M}+e^{j 2 \pi f_{\text {off }}\left[(q-1) T_{\mathrm{ss}}+\tau_{\mathrm{sam}}\right]} \xi \cdot Y\left(\tau_{\mathrm{sam}}\right) & q=8,9,10 \\
\eta_{q M}+e^{j 2 \pi f_{\text {off }}\left[(q-1) T_{\mathrm{ss}}+\tau_{\mathrm{sam}}\right]} \xi \cdot Z\left(\tau_{\mathrm{sam}}\right) & q=11\end{cases}
\end{aligned}
$$

where

$$
\begin{aligned}
Y\left(\tau_{\mathrm{sam}}\right)= & M \sum_{k=-26}^{26} \sum_{k^{\prime}=-26}^{26} B_{k} B_{k^{\prime}}^{*} e^{j 2 \pi k \Delta_{f} \tau_{\mathrm{sam}}} \\
& \times e^{j \pi\left[\left(k-k^{\prime}\right) \Delta_{f}+f_{\mathrm{off}}\right]\left(T_{\mathrm{ss}}+T_{\mathrm{sam}}\right)} \\
& \times \frac{\sin \mathrm{c}\left(\left[\left(k-k^{\prime}\right) \Delta_{f}+f_{\mathrm{off}}\right] T_{\mathrm{ss}}\right)}{\operatorname{sinc}\left(\left[\left(k-k^{\prime}\right) \Delta_{f}+f_{\mathrm{off}}\right] T_{\mathrm{sam}}\right)} \\
Z\left(\tau_{\mathrm{sam}}\right)= & M \sum_{k=-26}^{26} \sum_{k^{\prime}=-26}^{26} C_{k} B_{k^{\prime}}^{*} \\
& \times e^{j 2 \pi k \Delta_{f}\left(\tau_{\mathrm{sam}}-T_{G I 2}\right)} e^{j \pi\left[\left(k-k^{\prime}\right) \Delta_{f}+f_{\mathrm{off}}\right]\left(T_{\mathrm{ss}}+T_{\mathrm{sam}}\right)} \\
& \times \frac{\sin \mathrm{c}\left(\left[\left(k-k^{\prime}\right) \Delta_{f}+f_{\mathrm{off}}\right] T_{\mathrm{ss}}\right)}{\operatorname{sinc}\left(\left[\left(k-k^{\prime}\right) \Delta_{f}+f_{\mathrm{off}}\right] T_{\mathrm{sam}}\right)}
\end{aligned}
$$

and $^{4} \eta_{q M} \mathrm{~s}, q=8, \ldots, 11$, are independent, zero-mean, circularly symmetric complex-Gaussian random variables with variance

$$
E\left\{\eta_{q M} \eta_{q M}^{*}\right\}=\mathrm{SNR}^{-1} \gamma^{-1}
$$

In (16), the signal-to-noise ratio (SNR) is computed by SNR = $P /\left(N_{0} W\right)$, and

$$
\begin{aligned}
\gamma^{-1}= & \sum_{m=1}^{M} g_{m} g_{m}^{*} \\
= & M \sum_{k=-26}^{26} \sum_{k^{\prime}=-26}^{26} B_{k} B_{k^{\prime}}^{*} e^{j \pi\left(k-k^{\prime}\right) \Delta_{f}\left(T_{\mathrm{ss}}+T_{\mathrm{sam}}\right)} \\
& \times \frac{\operatorname{sinc}\left(\left(k-k^{\prime}\right) \Delta_{f} T_{\mathrm{ss}}\right)}{\sin \mathrm{c}\left(\left(k-k^{\prime}\right) \Delta_{f} T_{\mathrm{sam}}\right)} .
\end{aligned}
$$

${ }^{4}$ In the derivation, we make use of the facts that $4 \Delta_{f} T_{\mathrm{ss}}=1$ and $B_{k}=0$ for $k$ not a multiple of four.
For $\tau_{\text {sam }}>0$, the second subpreamble has a minor contribution to $\Xi_{10 M}$. We ignore this minor contribution in the derivation of (13) in order to keep the expression of $\Xi_{10 M}$ consistent with those of $\Xi_{8 M}$ and $\Xi_{9 M}$.

The probability of false alarm in a single comparison is defined as the probability that $\beta \mu_{9 M}>\left|\Xi_{9 M}\right|^{2}$ (or $\beta \mu_{10 M}>\left|\Xi_{10 M}\right|^{2}$ ). The probability of miss is the probability that $\beta \mu_{11 M} \leq\left|\Xi_{11 M}\right|^{2}$. It is difficult to characterize $\mu_{9 M}, \mu_{10 M}$, and $\mu_{11 M}$ since $\mu_{i}$, given by (10), is continuously updated. However, since $\mu_{i}$ is the maximum of correlator outputs' magnitude-square values that have been obtained before time $i$, we can approximate $\mu_{i}$ by the magnitude square of a correlation peak. Justification of this approximation will be done by checking the analytical result with simulation. Conditioned on $\tau_{\text {sam }}$ and $\xi$, the probability of false alarm in a single comparison $P_{\mathrm{fa}}(\cdot)$ and the probability of miss $P_{m}(\cdot)$ are given by

$$
\begin{aligned}
P_{\mathrm{fa}}\left(\tau_{\text {sam }}, \xi\right)= & \operatorname{Pr}\left\{\beta \mid \xi \cdot Y\left(\tau_{\text {sam }}\right)\right. \\
& \left.+\left.\eta_{\text {fa } 1}\right|^{2}>\left|\xi \cdot Y\left(\tau_{\text {sam }}\right)+\eta_{\text {fa } 2}\right|^{2}\right\}
\end{aligned}
$$

and

$$
\begin{aligned}
P_{m}\left(\tau_{\text {sam }}, \xi\right)= & \operatorname{Pr}\left\{\beta \mid \xi \cdot Y\left(\tau_{\text {sam }}\right)\right. \\
& \left.+\left.\eta_{m 1}\right|^{2}<\left|\xi \cdot Z\left(\tau_{\text {sam }}\right)+\eta_{m 2}\right|^{2}\right\}
\end{aligned}
$$

respectively, where $\eta_{\mathrm{fa} 1}, \eta_{\mathrm{fa} 2}, \eta_{m 1}$, and $\eta_{m 2}$ are independent zero-mean complex-Gaussian random variables with variance $\mathrm{SNR}^{-1} \gamma^{-1}$. It is desirable to express $P_{\mathrm{fa}}(\cdot)$ and $P_{m}(\cdot)$ as a function of $E_{\mathrm{ds}} / N_{0}$ where $E_{\mathrm{ds}}=(1 / 52) P T_{\mathrm{ds}}$ is the datasymbol energy, with $T_{\mathrm{ds}}=3.2 \mu \mathrm{s}$ being the duration of a data symbol excluding the cyclic prefix. Let

$$
\Omega=\gamma \cdot \mathrm{SNR}=\left(\frac{52 \gamma}{T_{\mathrm{ds}} W}\right) \cdot \frac{E_{\mathrm{ds}}}{N_{0}} .
$$

Also, let $2 \sigma_{\eta}^{2}=\Omega^{-1}$. Equations (18) and (19) can be expressed as

$$
\begin{aligned}
P_{\mathrm{fa}}\left(\tau_{\mathrm{sam}}, \xi\right)= & \operatorname{Pr}\left\{\beta>\mid \frac{\xi \cdot Y\left(\tau_{\mathrm{sam}}\right)}{\sigma_{\eta}}\right. \\
& \left.+\left.\frac{\eta_{\mathrm{fa} 2}}{\sigma_{\eta}}\right|^{2} /\left|\frac{\xi \cdot Y\left(\tau_{\mathrm{sam}}\right)}{\sigma_{\eta}}+\frac{\eta_{\mathrm{fa} 1}}{\sigma_{\eta}}\right|^{2}\right\}
\end{aligned}
$$

and

$$
\begin{aligned}
P_{m}\left(\tau_{\text {sam }}, \xi\right)= & \operatorname{Pr}\left\{\beta^{-1}>\mid \frac{\xi \cdot Y\left(\tau_{\text {sam }}\right)}{\sigma_{\eta}}\right. \\
& \left.+\left.\frac{\eta_{m 1}}{\sigma_{\eta}}\right|^{2} /\left|\frac{\xi \cdot Z\left(\tau_{\text {sam }}\right)}{\sigma_{\eta}}+\frac{\eta_{m 2}}{\sigma_{\eta}}\right|^{2}\right\}
\end{aligned}
$$

respectively. It is recognized that

$$
\begin{aligned}
& \left|\frac{\xi \cdot Y\left(\tau_{\text {sam }}\right)}{\sigma_{\eta}}+\frac{\eta_{\mathrm{fa} 2}}{\sigma_{\eta}}\right|^{2} /\left|\frac{\xi \cdot Y\left(\tau_{\mathrm{sam}}\right)}{\sigma_{\eta}}+\frac{\eta_{\mathrm{fa} 1}}{\sigma_{\eta}}\right|^{2} \sim F_{2,2}^{\prime \prime} \\
& \quad \times\left(2 \Omega v\left|Y\left(\tau_{\mathrm{sam}}\right)\right|^{2}, 2 \Omega v\left|Y\left(\tau_{\mathrm{sam}}\right)\right|^{2}\right)
\end{aligned}
$$

and

$$
\begin{aligned}
& \left|\frac{\xi \cdot Y\left(\tau_{\mathrm{sam}}\right)}{\sigma_{\eta}}+\frac{\eta_{m 1}}{\sigma_{\eta}}\right|^{2} /\left|\frac{\xi \cdot Z\left(\tau_{\mathrm{sam}}\right)}{\sigma_{\eta}}+\frac{\eta_{m 2}}{\sigma_{\eta}}\right|^{2} \sim F^{\prime \prime}{ }_{2,2} \\
& \quad \times\left(2 \Omega v\left|Y\left(\tau_{\mathrm{sam}}\right)\right|^{2}, 2 \Omega v\left|Z\left(\tau_{\mathrm{sam}}\right)\right|^{2}\right)
\end{aligned}
$$

where $\sim$ denotes "follows the distribution of" and $F^{\prime \prime}{ }_{2,2}\left(\lambda_{1}, \lambda_{2}\right)$ is the doubly noncentral $F$ distribution with 2,2 
degrees of freedom and noncentrality parameters $\lambda_{1}, \lambda_{2}$ [26]. It is known that [27]

$$
\begin{aligned}
\operatorname{Pr}\left\{f>F^{\prime \prime}{ }_{2,2}\left(\lambda_{1}, \lambda_{2}\right)\right\} & =Q\left(\sqrt{\frac{f \lambda_{2}}{1+f}}, \sqrt{\frac{\lambda_{1}}{1+f}}\right) \\
- & \frac{1}{1+f} e^{-\frac{f \lambda_{2}+\lambda_{1}}{2(1+f)}} I_{0}\left(\frac{\sqrt{f \lambda_{1} \lambda_{2}}}{1+f}\right)
\end{aligned}
$$

where $Q(\cdot, \cdot)$ is Marcum's $Q$ function and $I_{0}(\cdot)$ is the modified Bessel function of the first kind and order zero. Applying (23) and (24) to (21) and (22), respectively, and making use of the result of (25), we obtain closed-form expressions of $P_{\mathrm{fa}}\left(\tau_{\mathrm{sam}}, v\right)$ and $P_{m}\left(\tau_{\mathrm{sam}}, v\right)$, in which $v$ is related to $\xi$ by (4). The probability of synchronization failure $P_{\text {fail }}$ is, hence, given by

$$
P_{\text {fail }}=\frac{1}{T_{\mathrm{sam}}} \int_{-T_{\mathrm{sam}} / 2}^{T_{\mathrm{sam}} / 2} \int_{0}^{\infty} p(v) \times P_{\text {fail }}\left(\tau_{\mathrm{sam}}, v\right) d v d \tau_{\mathrm{sam}}
$$

where

$$
\begin{aligned}
P_{\text {fail }}\left(\tau_{\text {sam }}, v\right)=1- & {\left[1-P_{\mathrm{fa}}\left(\tau_{\mathrm{sam}}, v\right)\right]^{2} } \\
+ & {\left[1-P_{\mathrm{fa}}\left(\tau_{\mathrm{sam}}, v\right)\right]^{2} \cdot P_{m}\left(\tau_{\mathrm{sam}}, v\right) . }
\end{aligned}
$$

The term $1-\left[1-P_{\mathrm{fa}}\left(\tau_{\mathrm{sam}}, v\right)\right]^{2}$ is the probability that false alarm occurs at $n=9$ or $10 \mathrm{M}$. Note that in deriving (27), we make use of the fact that a miss cannot occur at $n=11 \mathrm{M}$ if there is already a false alarm at $n=9$ or $10 M$. Numerical computation of the double integration in (26) can be accomplished by a two-fold application of Simpson's rule.

\section{NumericAl Results AND DISCUSSION}

In most wireless LAN applications, access points and data terminal equipments are stationary. Measurement results have shown that the temporal variation of the signal fits a Rician distribution with a Rician factor ranging from 6 to $12 \mathrm{~dB}$ in one set of results [21] and from 3 to $7 \mathrm{~dB}$ in another measurement [23]. Recently, there is growing interest in the use of hand-held computing devices such as personal digital assistants. Using hand-held portable devices for communications in wireless LANs results in more severe fading. The spatial variation of the signal is primarily Rayleigh distributed or weakly Rician [22], [28]. Although the aforementioned results on spatial and temporal variations are specific to particular measurement sites and signal frequencies, the measured Rician factors serve as reference values for the present work. In this paper, we focus on the cases where the Rician factors are from $-\infty$ to $12 \mathrm{~dB}$.

\section{A. Comparison With Simulation Results}

Simulation was performed based on the synchronization model detailed in Section III. In the generation of each simulated $P_{\text {fail }}$ value, $10^{7}$ simulation runs were performed. In each run, we first generated a sequence of signal samples $\left\{r_{n}\right\}$ wherein the fractional time offset $\tau_{\text {sam }}$, the complex path gain $\xi$, and the complex-Gaussian noise samples $\left\{\eta\left(n T_{\text {sam }}+\tau_{\text {sam }}\right)\right\}$ were randomly generated according to their distribution functions. The frequency offset $f_{\text {off }}$ was fixed. Since the starting point of the timing-synchronization process is roughly at the end of the seventh short OFDM symbol (Section IV), $\left\{r_{n}\right\}$ was generated beginning from $n=7 \mathrm{M}+n^{\prime}$ where $n^{\prime}$ is the uncer-
TABLE I

Computed And Simulated $P_{\text {fail }}$ VAlues for Combinations of $f_{\text {off }}$ AND $\beta$ AT $E_{\mathrm{ds}} / N_{0}=20 \mathrm{~dB}$ AND $K=6 \mathrm{~dB}$

\begin{tabular}{rccc}
\hline$f_{\text {off }}(\mathrm{kHz})$ & $\beta$ & Computed $P_{\text {fail }}$ & Simulated $P_{\text {fail }}$ \\
\hline-210 & 0.25 & $5.97 \times 10^{-4}$ & $5.07 \times 10^{-4}$ \\
-90 & 0.25 & $5.51 \times 10^{-4}$ & $4.49 \times 10^{-4}$ \\
0 & 0.25 & $5.41 \times 10^{-4}$ & $4.59 \times 10^{-4}$ \\
90 & 0.25 & $5.49 \times 10^{-4}$ & $4.61 \times 10^{-4}$ \\
210 & 0.25 & $5.88 \times 10^{-4}$ & $5.19 \times 10^{-4}$ \\
\hline-210 & 0.50 & $0.90 \times 10^{-3}$ & $1.14 \times 10^{-3}$ \\
-90 & 0.50 & $0.91 \times 10^{-3}$ & $0.94 \times 10^{-3}$ \\
0 & 0.50 & $0.82 \times 10^{-3}$ & $1.04 \times 10^{-3}$ \\
90 & 0.50 & $0.84 \times 10^{-3}$ & $0.99 \times 10^{-3}$ \\
210 & 0.50 & $0.91 \times 10^{-3}$ & $1.16 \times 10^{-3}$ \\
\hline-210 & 0.75 & $0.82 \times 10^{-2}$ & $2.06 \times 10^{-2}$ \\
-90 & 0.75 & $0.74 \times 10^{-2}$ & $0.97 \times 10^{-2}$ \\
0 & 0.75 & $0.73 \times 10^{-2}$ & $1.70 \times 10^{-2}$ \\
90 & 0.75 & $0.75 \times 10^{-2}$ & $1.39 \times 10^{-2}$ \\
210 & 0.75 & $0.85 \times 10^{-2}$ & $1.72 \times 10^{-2}$ \\
\hline
\end{tabular}

tainty in the starting point. To model this uncertainty, one needs a statistical distribution of $n^{\prime}$, which varies for different receiver implementations and is usually not available. Here, we consider a simple case that this uncertainty is within $\pm M$ sampling instants and $n^{\prime}$ is uniformly distributed over $[-M, M]$. In each simulation run, $n^{\prime}$ was randomly generated according to this distribution. After $\left\{r_{n}\right\}$ was generated, $\left\{\Xi_{n}\right\}$ was computed by (7) and the synchronization procedure was performed. A synchronization failure was obtained if the declared time (in seconds) of the end of the first subpreamble was outside the range $8 \mu \mathrm{s} \pm 100 \mathrm{~ns}$, wherein $8 \mu \mathrm{s}$ is the length of the first subpreamble and a tolerance of $100 \mathrm{~ns}$ is sufficiently large to cover the time offset introduced by $\tau_{\text {sam }}$. The simulated $P_{\text {fail }}$ value is compared with the one calculated by (26).

Table I lists the computed and simulated $P_{\text {fail }}$ values for $f_{\text {off }} \in-210,-90,0,+90,+210 \mathrm{kHz}$ and $\beta \in 0.25,0.5,0.75$ at $E_{\mathrm{ds}} / N_{0}=20 \mathrm{~dB}$ and $K=6 \mathrm{~dB}$. For $\beta=0.25$ and $\beta=0.5$, the results indicate that the value of $P_{\text {fail }}$ predicted by (26) agrees with the simulated one with a percentage error less than $25 \%$, showing that the accuracy of (26) in predicting $P_{\text {fail }}$ can be considered acceptable. It is also apparent that the error of $P_{\text {fail }}$ estimated by (26) grows larger in the case of $\beta=0.75$ and the difference between the computed and simulated values is a factor of two to three. Despite this, it will soon be evident that the value of $\beta$ that is of practical significance is around 0.3 , so that one can still use (26) to predict $P_{\text {fail }}$ with acceptable accuracy. The accuracy for different fading conditions is also confirmed by the results shown in Fig. 2, which plots the computed and simulated values of $P_{\text {fail }}$ against $E_{\mathrm{ds}} / N_{0}$ for $f_{\text {off }}=0$ $\mathrm{kHz}$ and $\beta=0.3$ under the fading conditions $K \in-\infty, 6,12$ $\mathrm{dB}$. It is apparent that the computed $P_{\text {fail }}$ values are close to the simulated ones for $K=-\infty \mathrm{dB}$ and $6 \mathrm{~dB}$. Although the results are a little bit off for $K=12 \mathrm{~dB}$, the accuracy is still acceptable.

\section{B. Optimal Threshold Value}

Fig. 3 plots the $P_{\text {fail }}$ values against different choices of $\beta$ for $K \in-\infty, 6 \mathrm{~dB}, E_{\mathrm{ds}} / N_{0} \in 10,30 \mathrm{~dB}$, and $f_{\text {off }} \in 0,+232,-232 \mathrm{kHz}$. The optimal value of $\beta$ that mini- 


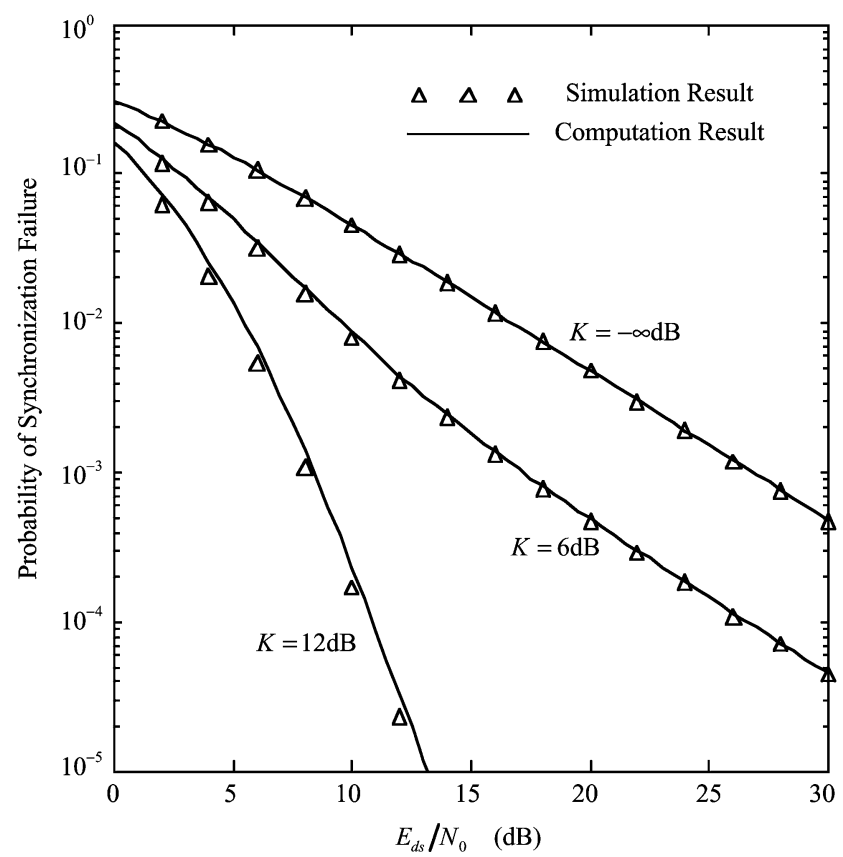

Fig. 2. Computed and simulated results of $P_{\text {fail }}\left(f_{\text {off }}=0 \mathrm{kHz}\right.$ and $\left.\beta=0.3\right)$.

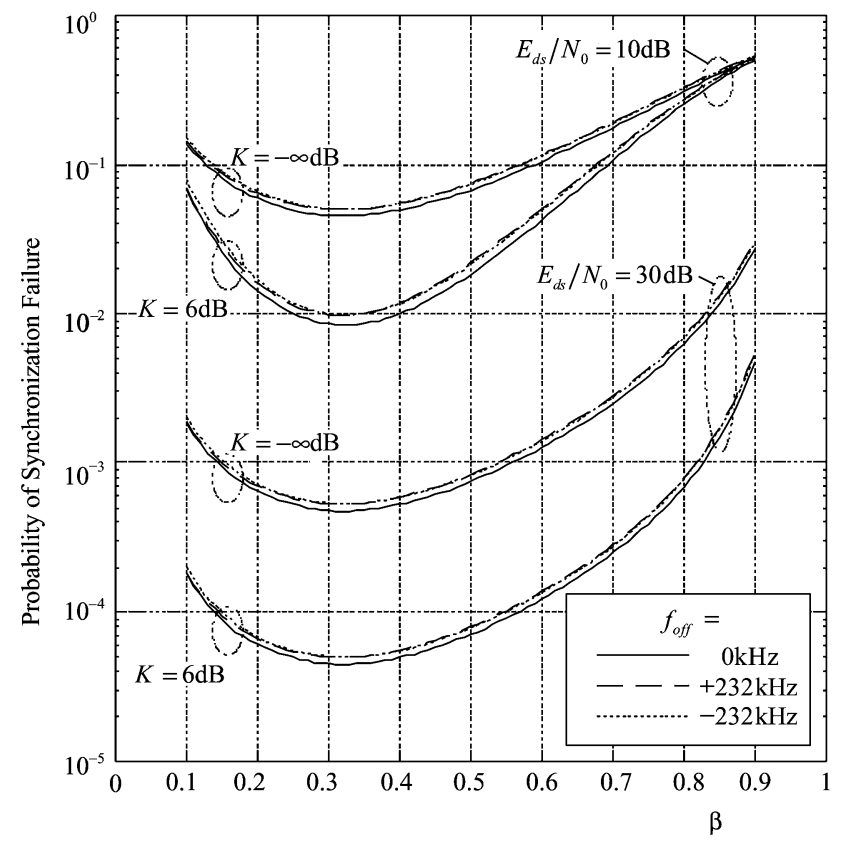

Fig. 3. $P_{\text {fail }}$ versus different choices of the threshold value $\beta$.

mizes $P_{\text {fail }}$ in all cases is $\beta=0.32$. This value of $\beta$ was used to generate the numerical results for the rest of paper.

\section{Effects of Frequency Offset}

The range of frequency offset we consider here is $\pm 232 \mathrm{kHz}$. Fig. 4 plots the $P_{\text {fail }}$ against $f_{\text {off }} \in[-232,232 \mathrm{kHz}]$ for $K \in\{-\infty, 6,12 \mathrm{~dB}\}$. For illustrative purpose, we consider $E_{\mathrm{ds}} / N_{0}=10 \mathrm{~dB}$. Although not shown here, it is also found that similar curves appear for other $E_{\mathrm{ds}} / N_{0}$ values. It is apparent that the difference of $P_{\text {fail }}$ values over the range of frequency offset is rather small. The presence of frequency offset has only

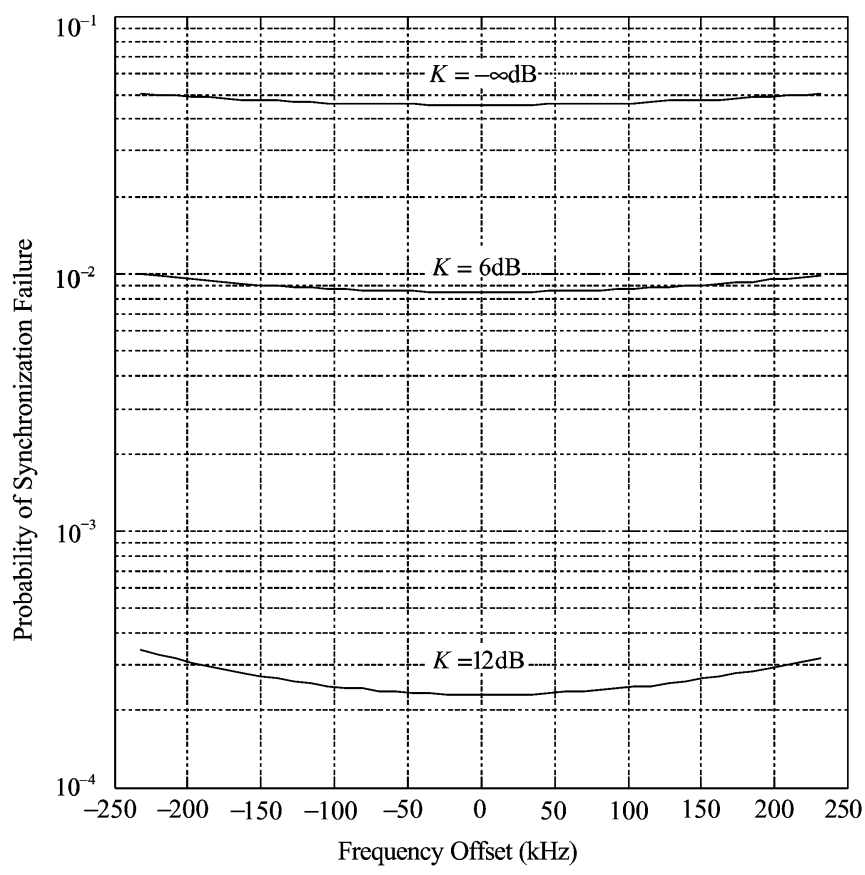

Fig. 4. Synchronization performance in the presence of frequency offset $\left(E_{\mathrm{ds}} / N_{0}=10 \mathrm{~dB}\right)$.

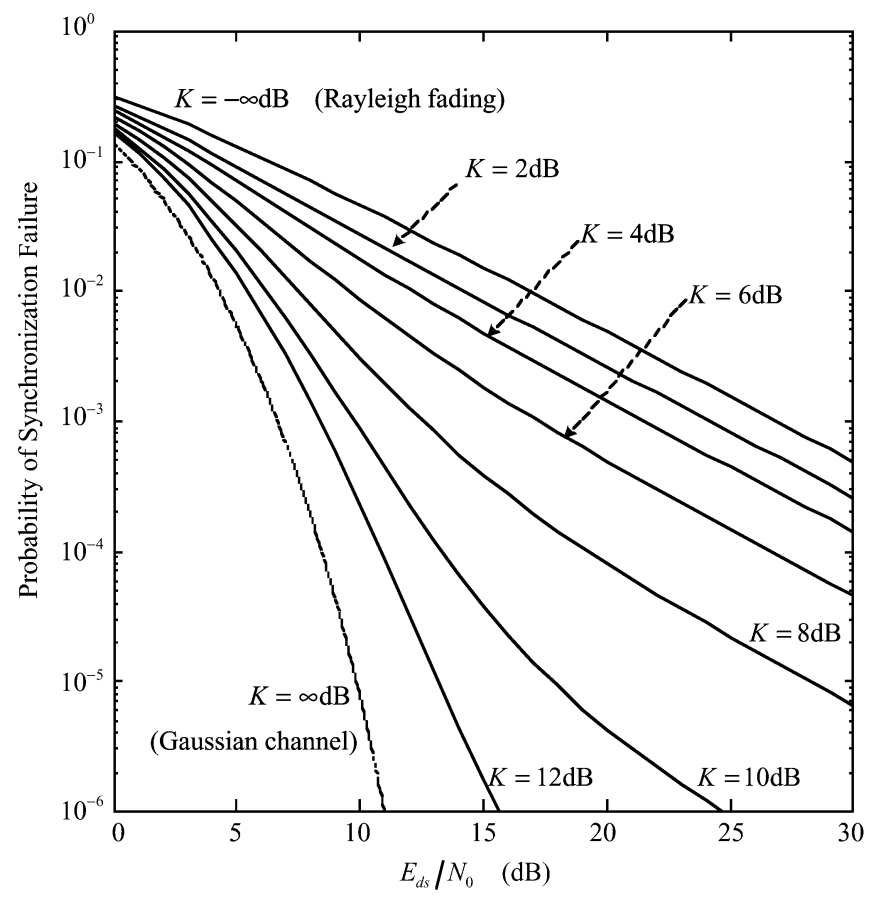

Fig. 5. Probability of synchronization failure for different Rician factors $K$.

a minor effect on $P_{\text {fail }}$. Therefore, $f_{\text {off }}=0 \mathrm{kHz}$ was assumed in the evaluation of $P_{\text {fail }}$ for the rest of paper.

\section{Different Fading Conditions}

In delay-insensitive data transmission such as file transfer, a loss of packets results in the need for retransmission so that its effect is to reduce the system throughput. The consequence of packet loss is more severe for multimedia data transmission, which is of growing importance in wireless LAN applications due to the popularity of the Internet. A packet loss rate of $1 \%$ 
TABLE II

\begin{tabular}{|c|c|c|}
\hline \multicolumn{3}{|c|}{$\begin{array}{r}\text { REQUIRED } E_{\mathrm{ds}} / N_{0} \text { TO ACHIEVE } P_{\text {fail }}=10^{-3} \text { AND } P \\
E_{d s} / N_{0}(\mathrm{~dB}) \text { at } P_{\text {fail }}=\end{array}$} \\
\hline$K(\mathrm{~dB})$ & $10^{-3}$ & $10^{-4}$ \\
\hline$-\infty$ & 26.8 & 36.9 \\
\hline 2 & 24.1 & 34.1 \\
\hline 4 & 21.5 & 31.4 \\
\hline 6 & 17.2 & 26.6 \\
\hline 8 & 12.5 & 19.2 \\
\hline 10 & 9.8 & 13.3 \\
\hline 12 & 8.4 & 10.9 \\
\hline$\infty$ & 6.7 & 8.5 \\
\hline
\end{tabular}

is generally considered the upper limit above which video and voice qualities become unacceptable [29], [30]. Since the probability of synchronization failure is a lower bound of the packet loss rate, we focus our attention to the cases that $P_{\text {fail }}=10^{-3}$ and $P_{\text {fail }}=10^{-4} .5$

Fig. 5 plots the $P_{\text {fail }}$ against $E_{\mathrm{ds}} / N_{0}$ for $K \in\{-\infty, 2,4, \ldots, 12 \mathrm{~dB}\}$. The curve for Gaussian channels $(K=\infty \mathrm{dB})$ is also plotted for reference. As expected, a higher Rician factor results in better synchronization performance. Table II lists the values of $E_{\mathrm{ds}} / N_{0}$ required to achieve $P_{\text {fail }}=10^{-3}$ and $P_{\text {fail }}=10^{-4}$ at different fading conditions. It is noted that relatively high $E_{\mathrm{ds}} / N_{0}$ values over $20 \mathrm{~dB}$ are generally required when $K \leq 6 \mathrm{~dB}$. In particular, the required $E_{\mathrm{ds}} / N_{0}$ values for achieving $P_{\text {fail }}=10^{-4}$ exceed 30 $\mathrm{dB}$ in the presence of Rayleigh or weakly Rician fading ( $K \leq 4$ $\mathrm{dB}$ ), which arises when portable computing devices are used. A higher transmission power, which is undesirable for portable devices where power saving is an important consideration, is therefore required.

\section{CONCLUSION}

We have derived the probability of synchronization failure for IEEE 802.11a wireless LANs on frequency-nonselective Rician fading channels. Based on numerical results, we have found that the optimal threshold value that minimizes $P_{\text {fail }}$ is $\beta=$ 0.32 . Computed results have indicated that for a frequency offset within the range $\pm 232 \mathrm{kHz}$, its effect on the synchronization performance is minor. We have also studied the impacts of different fading conditions on $P_{\text {fail }}$. The $E_{\mathrm{ds}} / N_{0}$ values required to achieve given $P_{\text {fail }}$ s have also been quantified. High $E_{\mathrm{ds}} / N_{0}$ values are generally required for fading conditions with low Rician factors.

\section{ACKNOWLEDGMENT}

The authors would like to thank the anonymous reviewers for their useful suggestions and comments which have led to considerable improvement in the manuscript.

\footnotetext{
${ }^{5}$ Although the packet loss rate is certainly more appropriate than the probability of synchronization failure in assessing the wireless LAN performance, the computation of packet loss rate involves consideration of the bit error rate, channel-estimation error, the probability of synchronization failure, etc., as well as the system load. Its computation is too involved and is beyond the scope of this paper.
}

\section{REFERENCES}

[1] IEEE Computer Soc., Dec., 301999.

[2] C. Bergljung and P. Karlsson, "Propagation characteristics for indoor broadband radio access networks in the $5 \mathrm{GHz}$ band," in Proc. IEEE PIMRC'98, Sept., 8-11 1998, pp. 612-616.

[3] J. Kivinen, X. Zhao, and P. Vainikainen, "Empirical characterization of wideband indoor radio channel at $5.3 \mathrm{GHz}$," IEEE J. Select. Areas Commun., vol. 49, pp. 1192-1203, Aug. 2001.

[4] I. Cuiñas and M. G. Sánchez, "Measuring, modeling and characterizing of indoor radio channel at $5.8 \mathrm{GHz}$," IEEE Trans. Veh. Technol., vol. 50, pp. 526-535, Mar. 2001.

[5] J. T. E. McDonnell, T. P. Spiller, and T. A. Wilkinson, "RMS delay spread in indoor LOS environments at $5.2 \mathrm{GHz}$," IEE Electron. Lett., vol. 34, pp. 1149-1150, May, 281998.

[6] T. S. Rappaport, Wireless Communications: Principles and Practice. Englewood Cliffs, NJ: Prentice-Hall, 1996.

[7] T. Saito, I. Tomoda, Y. Takabatake, K. Teramoto, and K. Fujimoto, "Wireless gateway for wireless home AV network and its implementation," IEEE Trans. Consumer Electron., vol. 47, pp. 496-501, Aug. 2001.

[8] K.-W. Yip, T.-S. Ng, and Y.-C. Wu, "Impacts of multipath fading on the timing synchronization of IEEE 802.11a wireless LANs," in Proc. ICC 2002, Apr. 28-May 2 2002, pp. 517-521.

[9] Y.-C. Wu, K.-W. Yip, and T.-S. Ng, "Design of multiplierless correlators for timing synchronization in IEEE 802.11a wireless local area networks," IEEE Trans. Consumer Electron., vol. 49, pp. 107-114, Feb. 2003.

[10] J.-J. van de Beek, M. Sandell, and P. O. Börjesson, "ML estimation of time and frequency offset in OFDM systems," IEEE Trans. Signal Processing, vol. 45, pp. 1800-1805, July 1997.

[11] N. Mochizuki, Y. Matsumoto, M. Mizoguchi, T. Onizawa, and M. Umehira, "A high performance frequency and timing synchronization technique for OFDM," in Proc. IEEE GLOBECOM'98, Nov., 8-12 1998, pp. 3443-3448.

[12] A. Palin and J. Rinne, "Enhanced symbol synchronization method for OFDM system in SFN channels," in Proc. IEEE GLOBECOM'98, Nov. 8-12, 1998, pp. 2788-2793.

[13] M.-H. Hsieh and C.-H. Wei, "A low-complexity frame synchronization and frequency offset compensation scheme for OFDM systems over fading channels," IEEE Trans. Veh. Technol., vol. 48, pp. 1596-1609, Sept. 1999.

[14] B. Yang, K. B. Letaief, R. S. Cheng, and Z. Cao, "Timing recovery for OFDM transmission," IEEE J. Select. Areas Commun., vol. 18, pp. 2278-2291, Nov. 2000.

[15] T. Keller, L. Piazzo, P. Mandarini, and L. Hanzo, "Orthogonal frequency division multiplex synchronization techniques for frequency-selective fading channels," IEEE J. Select. Areas Commun., vol. 19, pp. 999-1008, June 2001.

[16] T. M. Schmidl and D. C. Cox, "Robust frequency and timing synchronization for OFDM," IEEE Trans. Commun., vol. 45, pp. 1613-1621, Dec. 1997

[17] L. Házy and M. El-Tanany, "Synchronization of OFDM systems over frequency selective fading channels," in Proc. IEEE VTC'97, May, 4-7 1997, pp. 2094-2098.

[18] R. J. Castle, "A two-stage synchronization technique for OFDM," in Proc. IEEE VTC'99-Fall, Sept. 19-22, 1999, pp. 683-687.

[19] T. Wakutsu and M. Serizawa, "A carrier frequency offset and timing offset detection scheme for OFDM systems utilizing pilot sub-carriers," IEICE Trans. Commun., vol. E83-B, pp. 1854-1863, Aug. 2000.

[20] F. Tufvesson, M. Faulkner, P. Hoeher, and O. Edfors, "OFDM time and frequency synchronization by spread spectrum pilot technique," in Proc. IEEE Commun. Theory Mini Conf., June 6-10, 1999, pp. 115-119.

[21] R. J. C. Bultitude, "Measurement, characterization and modeling of indoor 800/900 MHz radio channels for digital communications," IEEE Commun. Mag., vol. 25, pp. 5-12, June 1987.

[22] T. S. Rappaport and C. D. McGillem, "UHF fading in factories," IEEE J. Select. Areas Commun., vol. 7, pp. 40-48, Jan. 1989.

[23] S.-C. Kim, H. L. Bertoni, and M. Stern, "Pulse propagation characteristics at $2.4 \mathrm{GHz}$ inside buildings," IEEE Trans. Veh. Technol., vol. 45 , pp. 579-592, Aug. 1996.

[24] P. H. Moose, "A technique for orthogonal frequency division multiplexing frequency offset correction," IEEE Trans. Commun., vol. 42, pp. 2908-2914, Oct. 1994. 
[25] J. G. Proakis, Digital Communications, 3rd ed. New York: McGrawHill, 1995.

[26] N. L. Johnson, S. Kotz, and N. Balakrishnan, Continuous Univariate Distributions, 2nd ed. New York, NY: Wiley, 1995, vol. 2, ch. ch. 30.

[27] R. Price, "Some noncentral F-distributions expressed in closed form," Biometrika, pt. 1, 2, vol. 51, pp. 107-122, June 1964.

[28] A. A. M. Saleh and R. A. Valenzuela, "A statistical model for indoor multipath propagation," IEEE J. Select. Areas Commun., vol. 5, pp. 128-137, Feb. 1987.

[29] M.-H. Fong and V. K. Bhargava, "Efficient integration of multi-rate traffic for 3rd generation DS-CDMA systems," in Proc. IEEE ICC'99, June, 6-10 1999, pp. 1426-1430.

[30] A. Zahedi and K. Pahlavan, "Capacity of a wireless LAN with voice and data services," IEEE Trans. Commun., vol. 48, pp. 1160-1170, July 2000.

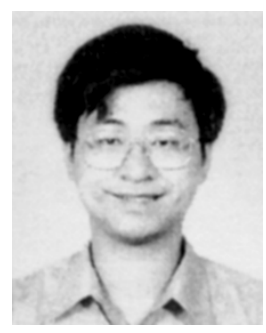

Kun-Wah Yip (M'96) received the B.Eng., (Hons.) and $\mathrm{Ph} . \mathrm{D}$. degrees in electrical engineering from The University of Bradford, Bradford, U.K., in 1991 and The University of Hong Kong, Hong Kong, in 1995, respectively.

From 1995 to 1998, he was a Research Associate and then a Postdoctoral Fellow at The University of Hong Kong. Currently, he is a Research Assistant Professor at the same university. He was also a Visiting Professor at Wireless Laboratories, NTT DoCoMo, in 2001. His research interests include WLANs, WPANs, communication circuits, spread-spectrum techniques, OFDM, and efficient simulation techniques for communication systems.

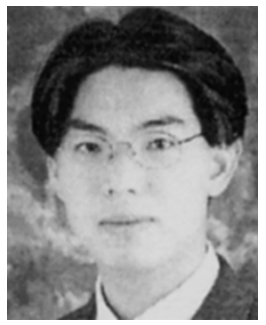

Yik-Chung Wu (M'02-S'03) received the B.Eng. (Hons) and M.Phil. degrees in electronic engineering from The University of Hong Kong, Hong Kong, in 1998 and 2001, respectively. Currently, he is working toward the Ph.D. degree at Texas A\&M University, College Station, TX.

In 2002, he was a Research Assistant at the University of Hong Kong. His research interests include digital signal processing with applications to communication systems and software radio and space-time processing.

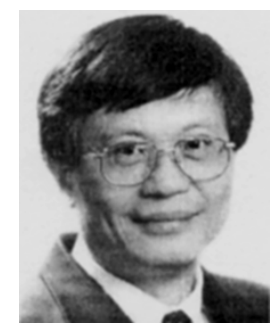

Tung-Sang Ng (S'74-M'78-SM'90-F'03) received the B.Sc. (Eng.) degree from the University of Hong Kong, Hong Kong, in 1972 and the M.Eng.Sc. and $\mathrm{Ph} . \mathrm{D}$. degrees from the University of Newcastle, Newcastle, Australia, in 1974 and 1977, respectively, all in electrical engineering.

He worked for BHP Steel International and The University of Wollongong, Australia, after graduation for 14 years and returned to Hong Kong in 1991 as Professor and Chair of Electronic Engineering. He has been Head of the Department of Electrical and Electronic Engineering since 2000. His current research interests include wireless communication systems, spread spectrum techniques, CDMA, and digital signal processing. He has published over 250 international journal and conference papers.

Dr. Ng was the General Chair of ISCAS'97 and the VP-Region 10 of IEEE CAS Society (1999 and 2000). He was an Executive Committee Member and a Board Member of the IEE Informatics Divisional Board (1999-2001) and was a member of IEE Council (1999-2001). He was awarded the Honorary Doctor of Engineering Degree by the University of Newcastle, Australia, in 1997 for his services to higher education generally and to engineering education specifically. He received the Senior Croucher Foundation Fellowship in 1999 and the IEEE Third Millennium medal in February 2000. He is a Fellow of HKIE. 